\title{
Horizons/Théâtre
}

Revue d'études théâtrales

10-11 | 2017

Genre et arts vivants

\section{Les variétés de la performance du genre}

\section{Shannon Jackson}

Traducteur : Chloé Déchery

\section{(2) OpenEdition}

\section{Journals}

Édition électronique

URL : http://journals.openedition.org/ht/472

DOI : $10.4000 /$ ht.472

ISSN : 2678-5420

\section{Éditeur}

Presses universitaires de Bordeaux

Édition imprimée

Date de publication : 1 juillet 2017

Pagination : 12-36

ISSN : 2261-4591

\section{Référence électronique}

Shannon Jackson, «Les variétés de la performance du genre », Horizons/Théâtre [En ligne], 10-11 | 2017, mis en ligne le 01 juillet 2018, consulté le 20 juillet 2019. URL : http:// journals.openedition.org/ht/472; DOI : 10.4000/ht.472

\section{(ब) $\odot \Theta \Theta$}

La revue Horizons/Théâtre est mise à disposition selon les termes de la Licence Creative Commons Attribution - Pas d'Utilisation Commerciale - Pas de Modification 4.0 International. 


\section{SHANNON JACKSON}

Shannon Jackson occupe la chaire Cyrus et Michelle Hadidi (Humanities) à I'University of California, Berkeley, où elle est professeure de rhétorique, théâtre, danse et performance studies. Elle est également associate vice chancellor for the arts and design travaillant à créer de nouvelles collaborations interartistiques entre départements, centres d'arts, initiatives de recherche, organismes de diffusion, programmes pédagogiques, sur l'ensemble du campus. Outre les nombreux articles et contributions à des catalogues de musée, elle publie plusieurs ouvrages personnels : The Builders Association: Performance and Media in Contemporary Theater, M.I.T. Press, en 2015, Social Works: Performing Art, Supporting Publics, chez Routledge en 2011, Lines of Activity: Performance, Historiography, and Hull-House Domesticity en 2000 et Professing Performance: Theatre in the Academy from Philology to Performativity en 2004. Elle reçoit au cours de sa carrière de nombreuses distinctions dont la John Simon Guggenheim Fellowship en 2015.

Du 12 au 14 décembre 2013 à Paris, Elizabeth Claire, historienne de la danse au Centre de recherches historiques (CNRS), Catherine Deutsch, historienne de la musique à Paris-Sorbonne, et Raphaëlle Doyon, historienne du théâtre à l'Université Paris 8, ont organisé un colloque international intitulé « Genre et création dans l'histoire des arts vivants $\gg$. Des chercheuses américaines reconnues pour leurs travaux sur le genre avaient chacune donné une conférence en session plénière : Lynn Garafola pour la danse, Suzanne Cusik pour la musique, et Shannon Jackson pour le théâtre et la performance.
Nous remercions les organisatrices ainsi que les structures et universités qui ont soutenu cet événement d'autoriser la présente publication du texte de Shannon Jackson. Nous avons souhaité conserver le contexte d'énonciation de cette conférence, notamment les allusions à une réflexion genrée transversale sur la musique (dès lors considérée comme un art vivant incarné), la danse, le théâtre et la performance. Nous remercions par ailleurs Shannon Jackson d'avoir revu la traduction française de son texte. 


\section{Les variétés de la performance du genre}

En 1894, Ellen Gates Starr, qui était membre d'une communauté de pionniers et une résidente de Hull House ${ }^{2}$, organisa une série de festivités pour des groupes d'enfants d'âges différents qui provenaient d'un quartier ouvrier et immigrant situé dans l'ouest de Chicago. Pendant une journée, elle prépara de la nourriture, disposa les chaises et arrangea les décorations sept fois de suite afin que chaque groupe puisse déguster les mets selon des plans de table différents et décorer le même arbre de Noël encore et encore. Elle décrivit ainsi sa journée à une amie : « cette semaine va être essentiellement consacrée à l'installation, la désinstallation et la ré-installation de décorations sur l'arbre de Noël. Demain, nous ferons la même chose trois fois de suite : d'abord les maternelles, puis les filles italiennes... et en soirée, les filles des magasins. » Starr ne cessa de s'adonner, pendant des heures, à une suite de mouvements répétitifs : se baisser, saisir et accrocher les décorations pour ensuite les décrocher de nouveau, se baisser, décorer, se lever, saisir et décrocher les décorations... une suite de mouvements qui s'inscrivait dans la pratique culturelle et réformatrice du settlement movement ${ }^{3}$.

En 1973, l'artiste Mierle Laderman Ukeles présenta une série de quinze performances simultanément à l'exposition de son travail dans plusieurs musées et galeries à travers les États-Unis. À l'occasion d'une de ces performances intitulée « Hartford Wash : Washing Tracks, Maintenance Inside », présentée au Wadsmorth Atheneum à Hartford dans le Connecticut, Ukeles se mit à quatre pattes pour laver les sols ainsi que les escaliers extérieurs du musée. Elle expliqua plus tard ce qui l'avait poussée à créer cette pièce : «L'entretien, ça use. Ça prend tout ton putain de temps (litt.) / On s'étourdit et on s'exaspère face à l'ennui. / La culture attribue un statut minable au travail d'entretien $=$ / salaires minimum, femmes au foyer $=$ non rémunérées $/$ Mais si je suis l'artiste, c'est moi qui dis ce qui est artistique. Et c'est ce que j'ai choisi de faire. » Ukeles ne cessa de s'adonner, pendant des heures, à une suite de mouvements répétitifs : se baisser, presser, frotter, se lever, jeter, se baisser, 
presser, frotter, se lever et jeter... une suite de mouvements qui s'inscrivait dans la pratique expérimentale et avant-gardiste d'un « art d'entretien ».

Ce qui m'intéresse avec ces pratiques performatives, c'est tant la façon dont elles se répondent que la façon dont elles restent sourdes l'une à l'autre. Toutes deux sont des indices d'un travail féminin et d'une certaine classe sociale. Toutes deux impliquent des gestes répétitifs ; toutes deux proposent une construction dynamique de l'espace ; toutes deux représentent la performance d'un travail genré qui est souvent invisible. Ces deux performeuses semblent travailler au nom de personnes et d'espaces qui ne coïncident pas avec ce qu'elles sont. Cependant et quelles que soient les caractéristiques thématiques et formelles qu'elles partagent, ces performances du genre s'inscrivent également dans deux histoires de thèmes et de disciplines qui demeurent très éloignées l'une de l'autre. La première performance date de la fin du XIX ${ }^{\mathrm{e}}$ siècle et prend place au sein du settlement movement, un mouvement américain qui encourage l'entreprenariat féminin et le développement de nouveaux processus créatifs au service des classes immigrantes et ouvrières. La deuxième performance date des années 1960, époque de gloire d'un art activiste qui émane, tout en s'y opposant, de pratiques issues de l'art conceptuel et qui célébraient un modèle masculiniste de créativité progressiste. En tant que telle, chacune de ces deux performances se situe également aux confins de ce que l'on pourrait appeler « les arts du spectacle », particulièrement si l'on considère que ces arts existent à travers des catégories distinctes telles que le théâtre, la danse et la musique. La première performance peut se lire comme une forme d'analyse de la performance de genre issue de la vie quotidienne. La seconde est davantage un exemple de « performance $a r t^{4} \gg$, ce qui soulève la question de savoir si le performance art est également un art du spectacle. On pourrait aussi déplacer la question par rapport au fait que nous sommes ici en France et se demander si une traduction littérale des « arts vivants » pourrait équivaloir, en anglais, au syntagme de « Live Art », une expression qui, en anglais britannique, témoigne d'une résistance par rapport aux arts du spectacle traditionnels. Mais je m'égare.

Laissez-moi revenir en arrière et faire part de ce que j'ai compris des enjeux d'un colloque, aussi large que celui-ci, sur le « Genre et les arts vivants ». En réalité, j'ai l'impression que ce rassemblement est déjà en lui-même assez radical et inhabituel en regard de la diversité des personnalités universitaires qu'il a su mobiliser (Marcia Citron, Suzanne Cusick, Lynn Garafola, Felicia Mc Carren). Alors que tant d'entre nous peuvent considérer leur travail comme étant de nature interdisciplinaire, nous participons le plus souvent à des réseaux universitaires nettement distincts les uns des autres. Beaucoup 
d'entre vous peuvent se rencontrer à des conférences d'histoire de la danse comme CORD [Congress on Research in Dance]; d'autres, à des sociétés universitaires de musicologues; certain.e.s d'entre nous se retrouvent à des conférences sur le théâtre comme la FIRT [Fédération Internationale des Recherches théâtrales] ou ASTR [American Society for Theatre Research]. Et peut-être que des chercheurs et chercheuses de ces réseaux seront aussi présent.e.s à une conférence sur les Performance Studies ${ }^{5}$, un de ces lieux où vous pourrez également me croiser. À mes yeux, ce rassemblement-ci diffère d'autres rassemblements propres aux Performance Studies du fait qu' il établit une distinction entre les différentes pratiques, techniques et histoires associées à des formes particulières, une spécificité qui tend à être oblitérée lorsque l'on se réfère à l'appellation générique de « performance ». Je me retrouve donc ici à écouter des panels ${ }^{6}$ de communications afin de découvrir comment des universitaires émanant de disciplines différentes voient, écoutent, touchent et interprètent ces pratiques particulières. Quelles histoires du geste les experts en danse sont-ils et elles capables de déceler dans la courbure d'un bras? Quand le son prend-il des résonances politiques pour un.e musicologue? Quelles normes les spécialistes en études théâtrales voient-ils ou elles contestées dans l'interruption d'une scène? Une conférence comme celle-ci oblige chacun.e d'entre nous à faire le tri entre ce que nous ne voyons et n'entendons pas et ce que les autres sont capables de voir et d'entendre. Cela nous oblige à reconnaître que chacun·e d'entre nous peut faire l'expérience de la même performance de façon tout à fait autre et y voir des références à des endroits que d'autres trouvent inintelligibles, des métaphores là où d'autres ne voient que du littéral, de la virtuosité là où d'autres voient de l'amateurisme ou trouver de l'« art » là où d'autres voient du « quotidien ». Les variétés de la performance impliquent ainsi des variétés de perception ainsi que des variétés de vocabulaire. Des rassemblements comme celui-ci nous obligent à reconnaître avec humilité que nous ne savons jamais ce que nous ne connaissons pas ; ce qui est toujours une bonne leçon pour tout.e universitaire, artiste ou citoyen.ne, quel que soit son genre.

Ce point m'amène à une autre série de questions et d'enjeux que je trouve opérants ici et qui émanent du constat que les analyses sur le genre sont tout aussi variées que les analyses sur la performance. Qu'est-ce que nous entendons en réalité par « genre » quand ce terme figure dans le titre d'un événement comme celui-ci ? Certains d'entre nous y voient un rapport analytique avec les politiques féministes spécifiques que les historien.ne·s ont réparties en « vagues » successives - première vague, seconde vague, troisième vague 
- et qui, d'après ce que Susan McClary a récemment observé, peuvent parfois créer une tendance à l'amnésie d'une génération à l'autre.

Au cours des années 1970 et 1980, les historiens entreprirent un vaste travail archéologique qui révéla des strates et des strates de mouvements féministes qui avaient été précédemment oubliés. Une tendance commença à apparaître : les nouvelles générations étaient contraintes de réinventer la roue - réinventer les mêmes arguments, les mêmes griefs, les mêmes stratégies pour s'organiser - et retombaient aussitôt dans l'oubli. D'un côté, c'était excitant de découvrir que nous faisions partie d'une lignée ininterrompue d'activistes. Mais, d'un autre côté, c'était aussi extrêmement déprimant. Pourquoi est-ce que ces percées avaient laissé si peu de traces derrière elles, même parmi les femmes ${ }^{7}$ ?

Cela entraîne plusieurs conséquences pour les questions du genre et de la performance. Tout d'abord, l'analyse met l'accent sur les mouvements féministes ; la recherche se concentre sur ces formes de comportements genrés qui cherchaient précisément à dépasser les restrictions fondées sur le genre. D'autres formes de performance du genre auraient certainement besoin d'être également historicisées mais les efforts se sont concentrés sur la question de l'activisme et une certaine forme de performance du genre. L'argument que met en avant McClary sur la nécessité pour chaque génération de devoir ré-inventer la roue nous rappelle la nécessité qu'il y a à continuer de raconter les histoires féministes. Certain.e.s d'entre nous qui ont découvert le féminisme il y a des décennies craignent peut-être qu'un tel enseignement soit daté et souhaiteraient traiter de sujets plus actuels. Mais je dois continuellement me rappeler à quel point le savoir de chaque nouvelle génération estudiantine demeure lacunaire. Des cours sur la performance féministe pourront me sembler convenus mais sembleront certainement très novateurs aux yeux des étudiant.e.s. Les étudiant.e.s n'auront pas accès à un savoir féministe tant que je ne leur propose pas de cours féministe. Mais nous pouvons aussi constater qu'il existe un lien entre la perte des « traces » d'un passé féministe et la perte des «traces » dans l'étude des représentations du passé plus généralement. En effet, les champs du genre et de la performance partagent une même difficulté méthodologique qui est que les recherches les plus intéressantes veulent avoir accès à ce qui est le plus éphémère.

Finalement, la citation de McClary fait du recouvrement des traces et de la restauration d'archéologies perdues des objectifs explicites des études de genre. Cette sorte d'entreprise reste fragile à un niveau logistique mais aussi à un niveau conceptuel alors que de nombreux universitaires débattent de la visée des analyses sur le genre. Ces débats ont grandement varié et ont parfois 
convergé à travers les champs de la musique, de la danse et du théâtre au sein des arts du spectacle. Aux États-Unis, j'ai remarqué que des numéros spéciaux ont été développés au sein de champs distincts et parfois, des revues universitaires ont été entièrement consacrées à la diversité des analyses sur le genre. Quand Sue-Ellen Case prit le poste de responsable éditorial de Theatre Journal, la revue devint un lieu fondamental pour le développement des débats féministes sur le genre et sur la sexualité - débats dont je proposerai la généalogie plus tard. Women and Performance couvrait un territoire plus large, de la performance expérimentale au chant et à la danse. Des numéros spéciaux de Dance Research Journal ont esquissé le programme prospectif des études féministes, au même titre que les livres et les essais polémiques - parfois les premières versions de textes qui seraient ré-écrits par la suite - d'Ann Daly, de Sally Banes, de Susan Foster, de Lynn Garafola, et de beaucoup d'autres. Le développement d'une revue comme Gender and Music a généré un contexte tout à fait bienvenu pour des recherches pointues menées par des personnalités comme Susan McClary, Carolyn Abbatte, Mary Ann Smart, ainsi que beaucoup, beaucoup d'autres recherches qui n'avaient pas besoin de défendre ou de rabattre les arguments féministes les plus connus et qui pouvaient simplement poser de nouvelles questions et développer de nouvelles méthodologies.

Quand je dis que les différents champs des études des arts vivants ${ }^{8}$ ont pu examiner des objets aussi divers que les objets promus par les études sur le genre, je veux dire que beaucoup de conversations qui s'en sont suivies ont eu à faire face aux mêmes complexités politiques et conceptuelles, complexités qui ont affecté ce que beaucoup de personnes croyaient vouloir entendre par « études de genre ». Il y a eu et il continue d'y avoir des tentatives pour réhabiliter les artistes femmes du monde du spectacle qui ont été exclues des histoires précédentes - l'effort consistant à montrer qu'il y a eu, en réalité, des femmes compositrices, des femmes interprètes de danse, des autrices de théâtre et des actrices-régisseuses dans le théâtre. De telles actions de recouvrement devinrent intéressantes à un niveau conceptuel quand elles commencèrent à altérer les récits officiels et à réévaluer ce qui fondait en première instance l'histoire des arts du spectacle. Actresses as Working Women ${ }^{9}$ de Tracy Davis nous invite à prendre au sérieux les formes de la culture populaire du $\mathrm{XIX}^{\mathrm{e}}$ siècle de la même façon que les analyses de Susan McClary sur Beyoncé contraignent les musicologues à prendre au sérieux les formes de musique populaire du début du $\mathrm{XXI}^{\mathrm{e}}$ siècle. La question du genre non seulement orientait les études des arts du spectacle vers d'autres artistes, mais également vers d'autres formes d'arts du spectacle. Ces différents sous-champs des études 
des arts du spectacle ont aussi été traversés par des changements conceptuels d'un autre type. Comme les organisateurs de la conférence l'ont révélé dans leurs choix de programmation, la théorie filmique s'est avérée être un terrain fondamental pour l'analyse de genre et a été adoptée avec enthousiasme dans le champ des arts vivants. L'impact de la « théorie du regard masculin ${ }^{10}$ » s'est ressenti à travers différents champs et a aligné les relations regardant/ regardé sur la relation homme/femme pour faire des interprètes féminins des objets qui étaient dépossédés d'eux-mêmes sur scène. Les réflexions d'Ann Daly sur la ballerine reproduisent les analyses théâtrales sur les actrices et les analyses musicologiques sur les chanteuses et les sopranes. La théorie du regard masculin s'est souvent attachée à définir le corps performant comme un corps dépossédé, une position féminisée - et, la plupart du temps, féminine. Comme le rappelle la fameuse citation de John Berger, « Les hommes regardent les femmes alors que les femmes s'observent en train d'être regardées ${ }^{11} \gg$. Cette théorie a également renforcé les relations hiérarchiques au sein du processus de création qui faisait de l'auteur/metteur en scène l'autorité masculine par rapport à l'interprète, une relation qui connaît son équivalent dans la relation hiérarchique tacite entre le chorégraphe et le danseur ou la danseuse, le compositeur, et l'interprète, chanteur ou chanteuse. Quand les féministes ont commencé à se douter que cette perspective ne permettait pas de rendre compte de la situation dans son ensemble, beaucoup ont commencé à s'intéresser à la complexité du corps de l'interprète et de l'événement spectaculaire. Ces retours sur la question de la complexité de l'acte spectaculaire, comme s'y sont essayés les travaux de Susan Foster en danse, la thèse de Raphaëlle Doyon sur les acteurs et actrices de l'Odin Teatret ou l'analyse musicologique, «Drastic or Gnostic ${ }^{12}$ ? », de Carolyn Abbatte, ont mis en évidence le fait qu'une très grande diversité d'activités créatrices devait être évaluée avant de pouvoir rendre le travail des femmes de nouveau visible.

Je dirais que les champs universitaires et artistiques du théâtre, de la danse et de la musique ont aussi connu une phase de croissance douloureuse lorsque la politique de l'inclusion et les questions philosophiques de méthode et de subjectivité ont commencé à faire débat. Aux États-Unis, on a souvent vu dans ce mouvement expansionniste la tentative de rendre compte d'un large éventail de positions identitaires et de relations différentielles - à travers les prismes des processus d'ethno-racialisation, de sexualités et de différenciation liés à l'âge et aux handicaps. Le récit de cette prénommée « politique identitaire » est lui aussi partiellement amnésique et a dû réinventer la roue plusieurs fois, passant sous silence les mouvements des droits civiques qui ont précédé la seconde vague du féminisme ainsi que les grands récits 
des cultures marginalisées avant que les universitaires féministes ne les redécouvrent. Mais la conséquence de tout cela, du moins dans le monde qui est le mien, est qu'il est tout à fait impossible de distinguer les questions de genre des autres marqueurs différentiels qui structurent nos vies. Renforcés par ces mouvements aussi bien que par les cadres proposés par le post-colonialisme et le transnationalisme, les départements des études de genre et des études féministes sont maintenant des lieux où l'on enseigne et où l'on étudie un ensemble nuancé de différences. Et l'on a souvent à cœur de ne pas imposer un modèle occidental d'autonomisation féministe sur des espaces transnationaux plus vastes, soucieux du fait qu'un modèle singulier de libération puisse fonctionner à la façon d'une forme involontaire d'oppression culturelle.

D'autres moments de croissance douloureux sont apparus au détour des complexes débats conceptuels qui ont porté sur la façon dont nous comprenions la subjectivité du genre en premier lieu. Si certaines études universitaires féministes pensaient remettre les femmes au premier plan, nous nous sommes vite retrouvé.e.s à nous demander comment nous pouvions savoir ce qu'était Une Femme en premier lieu. Je vais généraliser cette question de façon un peu réductrice et je renverrai au débat qui oppose essentialisme et anti-essentialisme. Nous savons qu'il existe des travaux nombreux et divers qui ont spéculé sur la possibilité d'une « esthétique féminine », d'une « expérience de femme », d'une « approche féminine de la connaissance » ou d'une « écriture féminine ». En effet, nous pouvons nous référer au lieu de notre rencontre, ici en France, pour rappeler aux Nord-Américain.e.s qui sont parmi nous que les tendances « essentialistes » qui proposent des généralités sur la Femme sont à l'origine des raisons pour lesquelles nous avons tout à la fois célébré et condamné le « féminisme à la française ». Bien que nous constations les retombées positives des travaux de Monique Wittig, d'Hélène Cixous et de Julia Kristeva, d'autres courants du débat sur le genre se sont plutôt concentrés sur la production discursive de catégories comme « l'expérience $\gg$ ou les binarités homme/femme ou sexe/genre. L'important essai de l'historienne Joan Wallach Scott, « Gender: a useful category of historical analysis ${ }^{13} \gg$, a ré-investi le concept $\mathrm{d}^{\prime} \ll$ expérience » afin d'empêcher une possible fixation autour d'un champ qui risquait de définir certaines sphères comme « pré-discursives » ou « éprouvées » qui aurait précisément empêché de penser comment de telles expériences éprouvées pouvaient être construites. En attendant, ma collègue Judith Butler est, bien sûr, la figure qui vient aussitôt à l'esprit pour avoir élaboré une réflexion anti-essentialiste - en particulier avec des textes comme Trouble dans le genre, Pour un féminisme de la subversion de l'identité ${ }^{14}$ et Ces corps qui comptent. De la matérialité 
et des limites discursives $d u$ sexe $\gg^{15}$. Pour cette dernière, comme pour Eve Sedgwick et beaucoup d'autres qui leur ont succédé, la perturbation discursive jouerait un rôle pivot dans la constitution d'une lecture queer du monde, une analyse qui distingue les analyses de genre des études déjà constituées sur les femmes et les identités gay et lesbiennes afin d'ouvrir un questionnement plus philosophique sur les différences fondamentales susceptibles d'exister au sein de toute catégorie. Ce qui est intéressant, c'est que les tendances anti-essentialistes d'une lecture queer ont également introduit un autre terme en $\ll \mathrm{p} \gg$ au sein des études des $\ll$ arts vivants $^{16} \gg$ [performing arts] ; le terme de « performativité » s'est ainsi imposé pour caractériser le genre comme une construction se constituant à travers une suite d'actes répétés qui contraignent ceux et celles qui les effectuent. Butler a démontré que ces actes performatifs construisent l'identité qu'ils prétendent représenter tout en exerçant l'emprise idéologique d'une identité de genre vécue «à la façon d'une croyance $\gg$. Comme j'espère pouvoir le développer un peu plus avant, la circulation d'un terme comme celui de « performativité » a tout à la fois stimulé et mis en péril les habitudes critiques de celles et ceux qui parmi nous avaient entrepris des analyses de genre au sein des études des arts vivants. D'un côté, le mot en -p semblait valoriser notre discipline. D'un autre côté, d'aucuns percevaient la critique anti-essentialiste comme une tentative de sape contre les efforts que nous avions déjà déployés pour poursuivre des recherches féministes. Comme McClary l'a dit dans une conférence récente et que je cite ci-dessous, nous avons eu l'impression que le vent qui avait fait gonfler les voiles du féminisme retombait au moment même où le bateau était prêt à partir. Pour ceux qui travaillaient sur la performance, la confusion était aggravée par la nature « expérientielle » des formes que nous étudions. Parce qu'il existe une tendance à placer les effets chorégraphiques, musicaux et théâtraux du côté de la sphère du non textuel, de l'actuel, du réel, de l'incarné, du présent, nous prenions apparemment le risque d'essentialiser nos formes - et, ce faisant, d'essentialiser la question du genre. En même temps, nous pouvions aussi dire - et c'est ce qu'ont fait certains d'entre nous - qu'il n'y avait pas de personne mieux placée que des chercheurs issus des études sur les arts vivants pour étudier la nature performative de l'expérience du genre. Qui, mieux que ceux qui savent comment se cultivent le geste, le son, l'émotion et le mouvement, peuvent comprendre l'acte de médiation que produit l'expérience corporelle et qui apparaît souvent comme un phénomène donné et naturel ?

Jusqu'à présent, je me suis concentrée sur l'étude de mouvements parallèles - du moins, c'est comme cela que je les comprends - au sein du dé- 
veloppement de la critique du genre dans les arts vivants. Pour ce qui suit, j'aimerais mettre en avant d'autres différences - politiques, méthodologiques et artistiques - qui ont altéré les relations parmi ceux d'entre nous qui étudient au sein de champs différents des arts vivants. Nous pourrions nous demander, par exemple, à quels endroits se manifestent les différences, les politiques, les tensions liées au genre parmi les trois formes de spectacle vivant que nous connaissons ? Est-ce qu'il y a une politique - et même une politique de genre - propre à chacune de ces trois formes de spectacle vivant et à la façon dont elles interprètent autrui ? Jusqu'à quel point le refus de toute théâtralité, disons, dans un récital de musique considéré comme sérieux, relève-til d'une question de genre en rapport avec les stéréotypes féminisés associés au théâtre ? Jusqu'à quel point le champ de la danse est-il déjà pris dans une hiérarchie de genre au sein des arts du spectacle vivant et est d'emblée considéré comme une activité « féminine » ? Jusqu’à quel point la tendance qui consiste à considérer la musique et le son comme des éléments d'arrière-plan au sein de l'expérience théâtrale est-elle une opération genrée qui force la musique à fonctionner à la façon d'une béquille au sein d'un processus de création théâtrale ?

Je reviendrai rapidement sur les exemples que j'ai utilisés en guise d'introduction, des exemples qui proviennent de mon propre parcours universitaire alors que j'étudiais la question du genre au sein de la performance du quotidien en vue de participer à la promotion d'une transformation sociale - et, plus récemment, alors que je poursuivais mes recherches sur cette chose qui revient souvent sous le nom de « performance art $\gg$. Le premier livre que j'ai écrit, Lines of Activity ${ }^{17}$, portait sur un mouvement pionner du $\mathrm{XIX}^{\mathrm{e}}$ siècle aux États-Unis au sein duquel les femmes (et, plus tard, les hommes) des classes moyennes et supérieures renonçaient aux liens des structures familiales traditionnelles afin de déménager dans les quartiers des classes immigrées et ouvrières. En formant une structure de parenté soudée, ces compagnons « pionniers » de la colonie étaient à la recherche d'une alternative radicale au modèle caritatif et travaillaient au sein de structures de gouvernance de quartier, de structures civiles, et, à terme, nationales, afin d'imposer des changements au nom de leurs « voisins » marginalisés. Entre les historien.ne.s féministes américain.e.s, il existe un grand nombre de débats sur les discours que les travailleuses des colonies ont déployé pour défendre leur mission. En partie afin de minimiser la critique qui visait inévitablement ces femmes célibataires travaillant « publiquement », les pionnières adoptaient souvent un discours maternel autour $\mathrm{du}$ « ménage civique $\gg$. Cette position fondamentalement « essentialiste » défendait la thèse selon laquelle ces femmes ne met- 
taient pas en péril le rôle maternel traditionnel mais qu'elles se contentaient d'élargir le rôle maternel et domestique de la « Femme » afin de toucher une sphère sociale plus large. Pour moi, ce discours soi-disant essentialiste est particulièrement intéressant quand on le place en contrepoint de leurs performances, de leurs actions et de leurs rituels quotidiens. Leurs comportements ne se contentaient pas de rejouer la maternité mais radicalisaient celle-ci au sein de lignées non-biologiques : leurs actions ne renforçaient pas seulement le rôle de la femme au sein du foyer, mais - grâce à des structures de vie communautaires, de nouveaux systèmes sociaux et des comportements répétés (à l'instar de l'arbre de Noël décoré et re-décoré) - réinventaient la notion de foyer en tant que telle. Il devint de plus en plus difficile de caractériser leur activité d'《essentialiste », en particulier si l'on prenait en compte la diversité des formes de leurs performances.

Passons rapidement de ce premier livre à un livre plus récent intitulé Social Works ${ }^{18}$ dans lequel figure Mierle Laderman Ukeles. Le plus souvent, on considère Ukeles comme une artiste de performance dont le travail repose sur des actions de longue durée et qui s'inscrit dans le champ des arts visuels ; une des nombreuses artistes féministes qui a réagi aux mouvements minimalistes et aux injonctions prescriptives de l'art radical issu des années 1960. Je vois dans son travail l'exemple intéressant d'une artiste qui a travaillé en réaction aux politiques du genre et à la façon dont celles-ci déterminent notre définition du processus de création. En tant qu'artiste visuelle travaillant de façon conceptuelle, elle se distingue par le choix qu'elle fait de développer cette critique à partir des structures de son œuvre. Après qu'Ukeles s'est mariée et a eu un enfant dans les années 1960, ses mentors lui annoncèrent que sa carrière était terminée. C'est en acceptant son statut en tant qu'épouse et en tant que mère qu'elle en vint à faire la critique de l'image masculiniste de l'artiste radical revendiquant son originalité et célébrant sa liberté et une indépendance sans entraves. Ce qu'elle a nommé ses « performances de l'entretien » étaient accompagnées d'expositions photographiques la montrant comme épouse et mère au foyer et faisant de la documentation de l'artiste en train d'habiller ses enfants, de laver leurs couches et de nettoyer la maison, une forme dévaluée d'un art du quotidien. La série « Washing, Tracks, Maintenance - Outside and Inside ${ }^{19} \gg$ montre également la dépendance, non seulement de la maisonnée, mais également de l'institution muséale par rapport aux formes quotidiennes d'un travail éprouvé selon les prismes du genre, de classe et des processus de racialisation.

J'en viens à me demander si une analyse sur le performance art ne pourrait pas être intégrée à notre étude des arts vivants, en grande partie parce que je 
crois que des artistes comme Ukeles ont beaucoup de choses à nous dire sur les politiques du genre qui ont animé le travail de création. Ukeles fait partie d'un groupe d'artistes pour qui les enjeux féministes exigeaient une transformation des formes et de leur processus de création : passer par la performance et créer des pièces très spécifiques et non orthodoxes était, pour elle, une façon de continuer de mener une action féministe.

Mais si mon travail féministe s'est autant intéressé au performance art et à la « performance de la vie quotidienne » qu'aux formes « des arts vivants », je réalise que je ne m'acquitterais pas de ma tâche ici aujourd'hui si je ne revenais pas sur la trajectoire spécifiquement «théâtrale » à travers l'analyse du genre au sein des « arts vivants ${ }^{20} »$ [en français dans le texte]. Sur ce sujet, l'on verra que la binarité entre essentialisme et anti-essentialisme est également effective, ce qui permet d'examiner en profondeur les enjeux conceptuels de la relation entre théâtre et performativité. Avant de rentrer plus avant dans les détails, il me semble cependant opportun de rappeler les mots d'avertissement prononcés par Judith Butler dont le travail figure de façon si proéminente au sein de ce débat.

L'institution d'un « objet propre » prend place, comme d'habitude, à travers une forme ordinaire de violence. Ainsi, on pourra interpréter des moments qui ont été fondateurs à un niveau méthodologique comme des actes systématiquement anti-historiques, des commencements qui ont contribué à fabriquer des récits d'auto-légitimation rétrospectifs, enterrant les complicités et les divisions sous l'image funèbre $d u \ll$ terreau $\gg^{21}$.

Mais si l'on tentait de clarifier la teneur de ces différents actes « anti-historiques » coupables de « complicité enterrée » en regard des questions de genre, de performativité et de représentation théâtrale ? Comme je l'ai relevé dans la première partie de ma communication, les efforts que nous déployons pour étudier le genre et les arts vivants sont complexifiés par la tentation que nous avons de construire les « objets propres » de ces deux domaines. Pour beaucoup d'entre nous, il n'y a pas d'équivalent entre l'étude de la performativité et l'étude des arts vivants. Aucun de ces deux champs d'études ne correspond non plus au champ des études théâtrales à proprement parler. De la même façon, pour beaucoup d'entre nous, il n'y a pas d'équivalence entre la théorie queer et les études lesbiennes et gay. Aucun de ces deux champs ne correspond non plus au champ des études féministes. Néanmoins, tous ces champs et ces termes peuvent être confondus les uns avec les autres comme c'est souvent le cas - même dans les discussions les plus prudentes. Parvenir à en découdre avec la trajectoire genrée de la représentation théâ- 
trale telle qu'elle s'est manifestée au sein des débats méthodologiques de la fin $\mathrm{du} \mathrm{Xx}^{\mathrm{e}}$ siècle exige ainsi de défaire une série d'équivalences hâtives et de déconstruire une série d'oppositions défensives. Les théories du genre et du théâtre semblent propulser les trajectoires critiques de chaque champ au sein d'une seule et même conversation pour les suspendre dans la conversation suivante. Chacun des deux termes peut être utilisé pour célébrer ou dénigrer l'autre terme.

Pour contribuer à la circulation des « objets propres » du genre et du théâtre, je vais proposer une thèse centrale qui concerne les opérations discursives de la « représentation théâtrale » tandis que nous discuterons plus avant des débats méthodologiques du $\mathrm{xx}^{\mathrm{e}}$ et du $\mathrm{XxI}^{\mathrm{e}}$ siècles. Selon moi, le théâtre fonctionne de façon omniprésente et contradictoire à cause de l'« essentialisme flexible » qui caractérise le syntagme. Le théâtre peut être utilisé comme une métaphore de la représentation, et peut, dans d'autres contextes, être convoqué comme une sorte de terreau de l'authentique. Alors que tout un courant de la pensée occidentale associe la théâtralité avec le figural, l’allégorique et la nature médiatisée de la représentation, un ensemble de théoriciens, dès la fin du $\mathrm{Xx}^{\mathrm{e}}$ siècle, utilisent des exemples théâtraux pour caractériser le littéral, le stable ou ce qu'on appelle, à partir d'un angle naïvement métaphysique, le « réel ». En d'autres termes, et selon les contextes, les convenances et les polémiques, le théâtre est tantôt renvoyé au côté essentialiste, tantôt renvoyé au côté anti-essentialiste d'un concept binaire. Par ailleurs, ce repositionnement constant n'est pas toujours réfléchi et, par conséquent, il n'est pas forcément «stratégique » et ne relève pas d'un essentialisme stratégique. Au contraire, cette flexibilité a tout autant été source de confusion qu'elle a été libératrice. Quand les études de genre (féministes, queer et autres) se sont intéressées à la question de l'essentialisme flexible de la théâtralité, cette combinaison s'est révélée à la fois utile et déroutante, en résonnance parfaite avec les luttes des théoriciennes féministes qui voulaient développer un projet politique tout en déconstruisant sa dimension essentialiste.

Afin de proposer un point de départ à partir duquel on pourrait élaborer une histoire intellectuelle, je vais discuter du travail de trois théoriciennes : Elin Diamond, Sue-Ellen Case et Judith Butler. Chacune de ces théoriciennes a grandement contribué à notre compréhension du théâtre et du genre tels qu' ils existent sous leurs diverses manifestations. De façon superficielle, ces trois théoriciennes pourraient être présentées comme les représentantes des trois grands courants des études de genre - Diamond est associée au « théâtre féministe », Case au «théâtre féministe lesbien » et Butler à la «performance queer ». Cependant, je voudrais également avancer que, bien 
qu'elle demeure utile à un niveau conceptuel, cette catégorisation rétroactive tend aussi à obscurcir les histoires importantes et les connexions signifiantes tant pour les études de genre que pour les études théâtrales.

De nouveaux problèmes ont surgi au sein de la théorie critique et féministe à la fin des années 1980, et Diamond, Case et Butler ont tenté de les résoudre de façon similaire. Comme je l'ai dit précédemment, les féministes avaient besoin de s'attaquer à la question de la sexualité et des enjeux philosophiques de ce que l'on a commodément appelé la pensée « poststructuraliste » ou anti-essentialiste. Alors que le problème de l'inclusion sexuelle et les problèmes liés au mouvement de déstabilisation anti-essentialiste n'étaient pas toujours reliés, à d'autres moments, ils étaient intimement liés l'un à l'autre. Suite aux travaux de Michel Foucault et aux évolutions de la psychanalyse et du déconstructionnisme, la sexualité s'est imposée comme un cadre conceptuel riche grâce auquel on pouvait théoriser l'hétérogénéité du sujet et les effets discursifs de façon plus générale. Pour certains, cependant, mettre l'accent sur $l^{\prime} \ll$ expérience féminine » ou l'« autonomie féminine » était nécessaire au féminisme : pour d'autres, bien sûr, cette emphase était naïve et relevait d'une conception réductrice du sujet féminin. Ainsi, au sein de cette configuration politique et épistémologique compliquée, on ne pouvait pas comprendre de façon très claire ce que cela signifiait que de mener des études de genre, d'être féministe ou de théoriser la sexualité.

Le texte d'Elin Diamond, «Brechtian Theory/Feminist Theory ${ }^{22}$ », propose une analyse visionnaire d'une pratique féministe qui rend compte de ce contexte critique complexe. La visée centrale de ce texte consiste en la fusion des objectifs féministes et de la technique théâtrale brechtienne. La référence à Brecht permet à Diamond de développer un argumentaire pour un théâtre conçu comme une pratique politique féministe. Une relecture créative des théories brechtiennes sur l'aliénation permet également de faire se correspondre le mouvement de déstabilisation de la pensée poststructuraliste et une pratique théâtrale adéquatement ironique. En même temps, la théorie théâtrale de Diamond insiste sur la nature « incarnée » du medium, « ancrant $\gg$ cette pratique hybride au sein d'une matérialité qui permet de lever toute suspicion anti-poststructuraliste. Le postulat d'un théâtre brechtien et féministe comme étant tout à la fois explicitement représentationnel et explicitement matériel, ironique et incarné, permet de développer une théorie essentialiste flexible qui est en phase avec les ruptures internes de la théorie féministe. 
Diamond déplace les concepts de Verfremdungseffekt, le « Non-pasMais $^{23} \gg$, et l'« historicisation » pour théoriser une Gestus féministe critique. À ce moment historique de la pensée féministe, mettre l'accent sur l'«expérience féminine » parait problématique. Un théâtre féministe qui se positionne au sein de ce paradigme expressif prend le risque de l'exclusion (de quelle-sfemme-s parle-t-on?) en présupposant une autorité et une authenticité expérientielles. Le processus de défamiliarisation lié au Verfremdungseffekt permet d'offrir un paradigme différent. Chercher à dé-familiariser le normal - afin de « transformer la chose qu'on veut faire comprendre, sur laquelle on veut attirer l'attention de chose banale, connue, immédiatement donnée, en une chose particulière, insolite, inattendue $\mathrm{e}^{24} \gg-$ pourrait contribuer à une entreprise de dé-normalisation de la masculinité et de la féminité. Plutôt que de considérer l'expérience féminine comme allant de soi, Diamond défend la thèse selon laquelle un théâtre de Gestus féministe pourrait rendre étrange la catégorie même de Femme. Le processus de défamiliarisation se poursuit à travers un processus $\mathrm{d}^{\prime}$ « historicisation $\gg$ brechtien, un mode de performance qui met en évidence la contextualité historique de la production du genre afin de la soumettre à une lecture critique. À mon sens, Diamond développe son argument théorique le plus novateur quand elle utilise le concept brechtien $\mathrm{du}$ « Non-pas-Mais » pour mettre en scène le caractère différentiel de la sexualité. Son analyse mérite d'être citée de façon extensive tant elle illustre un tournant épistémologique dans l'histoire de la théorie féministe.

La critique du genre, dans les pratiques artistiques et discursives, est souvent confondue, à tort, avec un autre topos de la théorie féministe : la différence sexuelle. Je voudrais avancer que l'expression de «différence sexuelle $\gg$ n'est pas synonyme d'une opposition entre les genres mais fait référence à l'existence d'un spectre de différences au sein de la sexualité. Je fonde en partie cette théorie sur le poststructuralisme qui privilégie la « différence »à travers tous les systèmes représentationnels, en particulier au sein du langage. La déconstruction derridienne postule la perturbation du signifié au sein du signe linguistique ou $d u$ mot : le mot apparemment stable est habité par un signifié qui porte les traces d'un mot qu'il n'est pas ${ }^{25}$.

Diamond trouve ainsi, au sein d'une discussion contemporaine sur la déconstruction, un langage qui résonne avec la dimension déstabilisatrice du théâtre brechtien. À partir d'une mise en relation avec la pensée brechtienne $\mathrm{du} \ll$ Non-pas-Mais », elle imagine un théâtre qui est capable de performer une forme d'auto-différencialité sexuelle. En renvoyant à la théorie de Derrida, la proposition de Diamond articule ainsi de façon explicite une mise 
en lien entre le poststructuralisme et la sexualité, mettant la pertinence du premier au service de l'hétérogénéité de la seconde. « La différence sexuelle peut alors être comprise comme une façon de déstabiliser les oppositions bipolaires qui constituent l'identité de genre ${ }^{26}$. $\gg$ Par ailleurs, Diamond nomme sa théorie une « critique du genre », écartant de façon explicite la possibilité d'associer de façon confuse sa théorie féministe avec la conception de la différence sexuelle comprise comme une «opposition de genre ». Pour elle, la nature différentielle de la sexualité était une question conçue sous l'impulsion de l'imaginaire et qui méritait d'être résolument explorée à travers ce qu'on appelle le féminisme - qui se distinguait sciemment d'une étude des « hommes » contre les « femmes ».

Enfin, le texte de Diamond renvoie à une pratique théâtrale en vue de défendre la viabilité politique d'une théâtralité féministe. L'entreprise de Diamond représente une attaque contre une vision du théâtre compris comme inutile ou comme « seul $»$ jeu.

Se souvenir de tels spectacles devrait nous rappeler la rigoureuse conscience de soi qui informe même le renversement de genres le plus ludique. Que la question du genre soit aliénée ou mise au premier plan, le spectateur est capable de lire un système de signes en tant que système de signes... le lexique du genre recouvre autant de pièges illusionnistes qui peuvent être exploités ou écartés à volontê2.

Diamond fait ainsi de la pratique théâtrale le lieu d'une rigueur réfléchie et d'une autonomie féminine ; un lieu où le genre est susceptible de se déployer « à volonté » comme un système de signes.

L'article de Sue-Ellen Case publié en 1989, « Towards a Butch-Femme Aesthetic $^{28} \gg$, correspond également à un moment critique dans la relation entre les études théâtrales et les politiques féministes. Le texte traite de l'impact du post-structuralisme - ou du « postmodernisme »-sur le féminisme. Il s'en prend à l'hétérosexime du mouvement féministe et développe un argumentaire sur le théâtre comme lieu privilégié d'un possible féministe et comme objet privilégié des analyses féministes. Il met également au premier plan le travail créatif de Split Britches, la compagnie de théâtre dont les performances au WOW Café de New York ont déclenché l'émergence de la théorie théâtrale féministe. Alors que Case s'inquiète des effets du «postmodernisme », postulant que son cadre « refuse au sujet son autonomie et son genre », en réalité, l'intuition d'une possibilité post-structurale stimule également la théorisation d'une esthétique «butch-femme ». Une critique post-structurale des discours binaires propose une conception de la sexualité comme un champ de différences, une hétérogénéité non reconnaissable au 
sein des polarités contraignantes de la « différence sexuelle ». Prenons, par exemple, ce passage souvent cité.

Ce ne sont pas des sujets divisés qui souffrent des vicissitudes d'une idéologie dominante. Il y a des êtres qui sont dans une relation de couple et qui ne s'empalent pas sur les pieux que sont la différence sexuelle ou les valeurs métaphysiques mais qui sont dans une constante danse de séduction avec le système de signes, et qui vagabondent, de flirts en inconséquences, jusqu'à connaître les doux attouchements de l'artifice, remplaçant ainsi le $\ll$ slash $^{29} \gg$ de Lacan par un bar lesbien ${ }^{30}$.

D’après ce modèle, être « divisé » peut signifier différentes choses. On peut être tourmenté par une division idéologique interne comme l'on peut être galvanisé par l'inconsistance et l'artificialité de cette même division. Autrement dit et comme je l'ai avancé précédemment, une critique post-structurale peut aller dans les deux sens. Alors qu'en théorie, elle court-circuite les revendications identitaires politisées, elle permet également de nourrir un projet d'expansion sexuelle. Par conséquent, la théorie que Case élabore à propos du travail de Split Britches peut également abonder dans les deux sens, évoquant tout à la fois ses « mauvaises » comme ses « bonnes » possibilités. Case utilise ainsi les termes de la représentation théâtrale - comme concept et comme pratique, comme norme et comme acte, comme cadre essentialiste et comme cadre non essentialiste - pour adopter le paradigme de la flexibilité qui joue à l'intersection de ces associations mouvantes.

Selon le scénario de la butch-femme analysé par Case, les processus de différenciation sexuelle sont rejoués mais aussi déjoués, en particulier à travers le concept de «mascarade » féminine. Plutôt que d'être le lieu de la normalisation féminine, la mascarade devient le lieu de la résistance féministe. Le texte utilise deux conceptions de la représentation théâtrale comme convention normalisée et action émancipatrice - pour les relier toutes deux à la notion de mascarade. De façon intéressante, le point de bascule entre ces deux conceptions repose sur la présence ou l'absence de conscientisation. Contrairement à la mascarade « féminine », la mascarade féministe « met au premier plan $\gg$, « affiche de façon explicite » et « joue délibérément » avec le genre ${ }^{31}$. Pour un mouvement féministe préoccupé par le lieu d'émergence de la puissance d'agir, la seconde signification qui pouvait être attribuée à une théâtralité consciente d'elle-même concordait parfaitement. Le langage de Case entrait en écho avec les formulations révolutionnaires du mouvement féministe. 
Ces rôles en tant que rôles octroient une puissance d'agir et une auto-détermination à un sujet historiquement passif et lui donnent au moins deux options en termes d'identification de genre, et, avec l'aide du camp, une ironie qui autorise une perception du sujet informée par une idéologie externe tout en donnant la possibilité au sujet d'adopter un rôle genré qui le fait apparaître comme s'il opérait de l'intérieur ${ }^{32}$.

En développant l'idée de cette distribution entre dedans et dehors, cette forme de théâtre féministe lesbien résolvait les complications politiques issues de la théorie poststructuraliste et féministe. Elle postulait un sujet doté d'une « perception » d'outsider ${ }^{33}$ mais aussi d'une « puissance d'agir » et d'un pouvoir d'《 auto-détermination » tout en reconnaissant son incarnation au sein de conventions sociales, de « rôles » genrés, et d' « apparences » sexuelles. En d'autres termes, sa subjectivité était divisée - une position anti-essentialiste - et, en même temps, ce même sujet était doté d'une volonté, d'une volition et maîtrisait sciemment ses performances - une position stratégiquement essentialiste. Comme chez Elin Diamond - les actrices féministes avaient la capacité d'adopter et d'abandonner différents rôles « à volonté ». Pour une lutte féministe qui souhaitait développer un projet politique tout en déconstruisant sa propre dimension essentialiste, l'essentialisme flexible du théâtre permettait au théâtre féministe lesbien de jouer des deux côtés.

Trouble dans le genre, Pour un féminisme de la subversion de l'identitée de Judith Butler, le texte que beaucoup ont associé avec l'avènement de la théorie queer, apparaît en 1990 pour tenter de régler un casse-tête similaire. Le premier paragraphe propose un résumé efficace de la pensée essentialiste féministe.

La pensée féministe a presque toujours tenu pour acquis qu'il existe une identité appréhendée à travers une catégorie de «femmes » qui non seulement introduit les intérêts et les buts féministes dans le discours, mais qui définit également le sujet pour lequel la représentation politique est recherchée $e^{35}$.

Le texte de Butler renvoie à Michel Foucault et à Jacques Derrida dans ses deux premières notes de bas de page, et dès la deuxième page, réfute « l'ensemble » des préjugés de la théorie féministe à partir de l'argument paradigmatique de la théorie post-structuraliste et de ses implications radicalement anti-essentialiste.

Foucault signale que les systèmes juridiques du pouvoir produisent les sujets $q u$ 'ils viennent ensuite à représenter. [...] Si cette analyse se révèle correcte, la formation juridique du langage et de la politique représentant les femmes comme 
le « sujet 》 du féminisme est alors elle-même une formation discursive et l'effet non moins discursif d'une certaine version de la politique de représentation ${ }^{36}$.

Butler s'en prend alors à la notion d'une identité, d'une psyché ou d'un langage féminin « d'avant » qui pourraient exister, d'une manière ou d'une autre, avant ou au-delà des opérations produites par les normes discursives. Le reste de Trouble dans le genre est consacré aux implications de ces remarques préliminaires. Butler ré-examine et re-situe des arguments clefs au sein de la théorie féministe critique et sociale en évaluant de façon minutieuse et en ré-intégrant les théories post-structuralistes sur les questions de l'identité et de la représentation. Tandis que la théorie des actes de langage de J.L. Austin n'est pas explicitement citée dans Trouble dans le genre, la définition qu'Austin donne de la performativité apparaît à plusieurs endroits du texte. La notion que le langage puisse être constitutif plutôt qu'expressif entre parfaitement en écho avec sa thèse selon laquelle le discours a le pouvoir de produire ce qu'il se contente de décrire. En travaillant à partir de l'idée anti-essentialiste de la Femme comme « effet » discursif, à l'encontre de l'idée d'une conscience féminine existant « avant la loi », le texte de Butler défend le principe que l'identité féminine émerge à partir d'un processus qui, en même temps, la nie.

Les derniers chapitres de Trouble dans le genre tentent de considérer la signification de ces arguments par rapport à la question de l'activisme. Ce sont les pages qui ont été le plus souvent lues, retenues, mésinterprétées et débattues. Ces pages ont également stimulé et troublé les statuts du théâtre et de la performance, comme termes et comme pratiques, tout au long de la décennie suivante. Étant donné le fait que le sujet, quel que soit son genre, est constamment enchevêtré et toujours en train de se référer aux normes du genre, les notions conventionnelles d'action libératoire et d'altérité subversive ne pouvaient pas être pensées à partir du cadre proposé par Butler. Celle-ci a ainsi promu la performance de la « parodie de genre $\gg$ comme une manière d'évoquer de façon différente la normalisation du genre. « La question n'est pas de savoir s'il faut ou non répéter, mais comment le faire. Il s'agit dès lors de répéter en proliférant radicalement le genre, et ainsi de déstabiliser les normes du genre qui soutiennent la répétition ${ }^{37}$. $\gg$ Alors que le langage a pu apparaître familièrement théâtral, il existe une distinction particulière au sein de l'expression de « temporalité complexe » que théorise Butler et selon laquelle ni l'acteur ou actrice ni les normes de genre ne pré-existent les un.e.s aux autres. La parodie est, pour Butler, une façon d'imaginer une pratique politique au sein de ce qui n'a de cesse d'être un processus récurrent, c'est-àdire l'ensemble des moyens performatifs à travers lesquels la représentation 
impose un genre qu'elle fait mine de décrire. Enfin, si les théories queer de la performativité ont émergé en même temps que la critique anti-essentialiste du genre, je souhaiterais avancer qu'elles ont aussi entrainé la formulation d'hypothèses confuses sur les questions des frontières épistémologiques, des « complicités enterrées » et des parentés intellectuelles qui continuent de nous troubler aujourd' hui.

Je pense que nous pouvons constater à quel point ces complications se sont manifestées au sein du débat entre théâtre et performativité, notamment à partir de la question du « choix » ou de la «volonté » dans les opérations performatives. L'extrapolation de la pensée de Derrida que propose l'articulation de Butler entre le féminisme et la théorie des actes de langage met en avant l'absence d'une conscience pré-juridique qui pourrait émettre une intention et une volition avant et en dehors de la loi. L'absence d'un tel état pré-conscient ou d'une identité « d'avant » était constitutive, chez Butler, du développement de sa critique du féminisme, de sa critique de l'essentialisme, et de sa théorie de la performativité. Au milieu du ravissement et de la fureur qui suivirent la sortie de Trouble dans le genre, cependant, la mésinterprétation la plus commune portait sur l'existence d'un acteur ou d'une actrice consciemment performant.e qui aurait été un préalable à la performance du genre. Butler prit soin, au cours de nombreuses discussions, de tâcher de corriger cette méconception. L'insistance dont elle témoignait ne fit qu'entrainer davantage de problèmes. Après tout, l'hypothèse d'une performance féministe consciente d'elle-même était au cœur de la théorisation de plusieurs formes d'activisme féministes et sexuelles depuis le début des années 1990. On se souviendra que, pour Sue-Ellen Case, la présence d'une intentionnalité distinguait la mascarade féminine de la mascarade féministe. Dans les publications qui suivirent, Case demeura suspecte par rapport à la théorie de Butler et finit par accuser son modèle théorique de fonctionner à la façon d'une métaphysique inversée au sein de laquelle les normes juridiques occuperaient la fonction grammaticale d'un principe primaire et autogénérateur au lieu d'occuper la place d'un agent conscient de $\operatorname{soi}^{38}$. De même pour Elin Diamond, la Gestus féministe exigeait une « rigoureuse conscience de soi $\gg$ qui était susceptible de révéler le caractère illusoire du genre comme étant quelque chose qui pouvait être « endossé ou écarté à volonté $^{39} \gg$. Quand une nouvelle version de son essai parut dans Unmaking Mimesis $^{40}$, Diamond questionna cette tension. Elle était davantage encline à accepter la théorie de Butler d'une «temporalité complexe » au sein de laquelle ni les normes ni la conscience de soi de l'acteur ou de l'actrice ne précédaient les unes aux autres. Elle corrigea également certaines de ses 
phrases pour rendre compte d'un nouveau contexte discursif au sein duquel le vêtement ne faisait certainement pas la femme. Au lieu de « le genre est révélé à la façon d'un costume sexuel ${ }^{41}$ », elle avança que le « genre est constamment révélé en tant que "performativité", en tant que système de normes juridiques que le sujet évoque afin d'exister culturellement ${ }^{42} \gg$. Diamond retira également la référence à la capacité qu'a l'acteur ou l'actrice de se débarrasser « à volonté » du caractère illusoire du genre et proposa à la place un long développement sur l'impact de la performativité queer sur la théâtralité féministe. Son argument le plus convaincant devint une revendication disciplinaire. Tandis qu' elle reconnaissait que la thèse de Butler selon laquelle la performance théâtrale «implique une autre performance qui, à un niveau ontologique, précède et fabrique les effets du genre... [était] irréfutable $\gg$, elle suggérait également que cette thèse faisait l'apologie d'une insularité théorique.

La performance, comme je l'ai déjà écrit ailleurs, est le lieu au sein duquel la performativité se matérialise sous une forme concentrée, où les « conventions cachées ou dissimulées 》 d'actions qui ne sont que des répétitions peuvent être explorées et réinventées ${ }^{43}$.

Il y a tant à dire sur cet entrelacs de débats, mais j'espère à présent que nous pouvons faire un pas de côté et concevoir de façon plus abstraite les implications de tels débats. Délimiter les contours des « objets propres » des arts vivants est une tâche ardue, tant en raison de l'essentialisme flexible qui caractérise les arts vivants qu'en raison des contextes relationnels mouvants au sein desquels nous théorisons la performance. De plus, il y a des raisons liées au genre qui expliquent pourquoi les débats sur le théâtre se sont révélés si difficiles entre la fin du $\mathrm{Xx}^{\mathrm{e}}$ et le début du $\mathrm{XxI}^{\mathrm{e}}$ siècle. La question de la sexualité - et plus particulièrement d'une sexualité qui était révélée comme le marqueur auto-différentiel de l'identité - a été une partie intrinsèque de l'histoire du féminisme. L'auto-différentialité de l'identité était également partie intégrante de la conversation autour du théâtre féministe. Souvenez-vous d'Elin Diamond qui théorisa le « Non-pas-Mais » de la sexualité déconstruite au sein de ce qu'elle appela le discours du théâtre féministe. Plus tard, on associera une telle structure de pensée à la performativité queer - parfois à l'exclusion des théories du féminisme et de la représentation théâtrale. Le fait que de tels liens entre la pensée du féminisme et la pensée du théâtre aient pu être oblitérés, même si cela ne se fit qu'au sein de quelques années de contemplation théoriques queer, rend compte de notre tendance à l'amnésie disciplinaire ainsi que d'une distribution inégale du pouvoir institutionnel. Si Susan 
Mc Clary s'inquiétait de notre capacité à oublier les mouvements féministes d'une génération à l'autre, ce court récit sur les statuts du genre, du théâtre et de la performativité montre que de tels oublis peuvent survenir au sein d'une même génération. Je me demande combien d'oublis, de projections et de méconnaissances au sein d'autres champs des arts vivants et spectaculaires certain.e.s d'entre vous ont déjà rencontrés ou risquent de rencontrer au cours de leurs recherches. C'est seulement en reconnaissant ces distinctions - dans des rencontres aussi rares qu'elles sont nécessaires, à l'instar de ce colloque - que nous pouvons cultiver un respect pour les approches variées de la performance et célébrer les connexions encore inexplorées au sein de la diversité des performances de genre qui s'impose à nous tou.te.s.

Traduction Chloé Déchery

(Maîtresse de conférences, Études théâtrales, Université Paris 8)

\section{Notes}

1. Le GIS Institut du genre, l'IEC (GID-Ile de France), le CRH (UMR 8558 CNRSEHESS), le LabEx Cap, HéSam, le RING, l'Université Paris-Sorbonne, le CREIM, le CRAL (UMR 8566 CNRS-EHESS), PLM, et le Ministère de la culture et de la communication Fonds Pascal.

2. Note de la traductrice : Hull House est un centre d'œuvres sociales cofondé par Jane Addams et Ellen Gates Starr à Chicago en 1889 afin de promouvoir des actions sociales et réformatrices auprès des habitants du quartier largement issus de l'immigration et de milieux ouvriers.

3. Note de la traductrice : le settlement movement est un mouvement social libéral qui connaît son apogée autour des années 1920 en Angleterre et aux États-Unis. Lobjectif du mouvement consiste à réunir les membres des classes les plus aisées avec ceux de classes plus défavorisées pour vivre plus de façon harmonieuse dans une communauté solidaire issue de la société civile. Le principal but de ce mouvement est la création de «maisons d'accueil » dans les zones urbaines pauvres des grandes villes dans lesquelles des bénévoles issus des classes moyennes et aisées vivent dans l'espoir de partager de favoriser un plus grand accès au savoir et à la culture et d'atténuer la pauvreté de leurs voisins à plus faibles revenus. En 1913 aux États-Unis, il y a 413 implantations dans 32 États. Fondée par Jane Addams à Chicago, Hull House est la deuxième « maison d'accueil ", à la suite de Toynbee Hall créé dans l'Est de Londres.

4. Note de la traductrice : l'appellation anglaise "performance art », conformément aux règles d'usage, est laissée telle quelle et n'est pas signalée en italique qu'elle puisqu'elle recouvre une tradition historique, culturelle, artistique et disciplinaire spécifiquement 
nord-américaine (et qui serait l'équivalent de ce que l'on appelle, en Grande-Bretagne, le "Live Art", par exemple).

5. Note de la traductrice : le champ des "Performance Studies ", à l'origine fondé aux États-Unis, notamment au sein des départements de New York University et de NorthWestern University à Chicago, n'a pas d'équivalent en langue française et ne correspond pas exactement, par exemple, au champ des études théâtrales. L'expression est donc laissée telle quelle dans le texte.

6. Note de la traductrice : Le terme anglais de «panel » est laissé tel quel dans le texte car il permet de faire référence au découpage par spécialisation quautorise ce mode d'organisation des communications au sein d'événements universitaires (colloques ou journées d'étude).

7. Susan McClary, «Making waves: opening keynote for the twentieth anniversary of the Feminist Theory and Music Conference », Women E Music, vol. 16, 2012, p. 1.

8. Note de la traductrice : tandis que l'expression "performing art » au singulier est conservée telle quelle dans le texte, l'expression au pluriel de "performing arts " est tantôt traduite par l'expression française d'" arts du spectacle », lorsque l'autrice réfère aux trois champs les plus repérables des arts du spectacle : théâtre, danse, musique ; tantôt traduite par l'expression française d'" arts vivants » qui non seulement renvoie au titre du colloque dans lequel le texte de la communication fut présenté originellement mais qui permet aussi de rendre compte et d'intégrer l'étude des comportements, des pratiques de soi et des mises en scène de la vie quotidienne au sein du champ d'étude de la performance comprise selon son acception anthropologique.

9. Louvrage est publié sous le titre Actresses as Working Women: Their Social Identity in Victorian Culture chez Routledge (1991). Le titre de l'ouvrage, non traduit en langue française, pourrait être traduit par «Les actrices, femmes du monde du travail, et leur identité sociale dans la culture victorienne ».

10. Note de la traductrice : la «théorie du regard masculin », autrement appelée en anglais «Male Gaze Theory » est une théorie qui a été élaborée par l'universitaire britannique féministe Laura Mulvey et plus tard redéveloppé dans son ouvrage sur le cinéma, Visual Pleasure and Narrative Cinema, publié en 1998 et qui a fait autorité non seulement dans les études de cinéma mais également dans les arts plastiques, l'histoire de l'art, les études de spectacle vivant et les arts vivants. Voir Laura Mulvey, «Visual Pleasure and narrative cinema ", Screen, Volume 16, Issue 3, 1 October 1975, p. 6-18. Article traduit partiellement dans Ginette Vincendeau et Bérénice Reynaud (dir.), Vingt ans de théorie féministe sur le cinéma, CinémaAction n ${ }^{\circ}$ 77, 1993, p. 17-23 puis en version intégrale : «Plaisir visuel et cinéma narratif », traduction Gabrielle Hardy, Débordements, 20 février 2012, article consulté en ligne le 31 août 2017 à l'adresse : http://www.debordements.fr/Plaisir-visuel-et-cinema-narratif. Laura Mulvey, Visual and other pleasures (2 édition), Houndmills, Basingstoke, New York, Palgrave Macmillan, 2009 [1989].

11. John Berger, Voir le voir [1972], traduit par Monique Triomphe, Paris, Alain Moreau, 1976, p. 51.

12. Carolyn Abbate, "Music - Drastic or Gnotic? ", dans Critical Inquiry, The University of Chicago Press, vol. 30, n 3, Printemps 2004, p 505-536.

13. Traduit en français sous le titre "Genre : une catégorie utile d'analyse historique ", traduit par Eleni Varikas, dans Le Genre de l'histoire, sous la direction de Christine 
Planté, Michèle Riot-Sarcey et Eleni Varikas, Les Cahiers du Grif n 37-38, 1988, p. $125-153$.

14. Judith Butler, Gender Trouble: Feminism and the Subversion of Identity, Londres et New-York, Routledge, 1990. Publié en français sous le titre Trouble dans le genre, Pour un féminisme de la subversion de l'identité, traduit par Cynthia Kraus, Paris, La Découverte, 2005.

15. Judith Butler, Bodies That Matter, The Discursive Limits of Sex, Londres et New York, Routledge, 1993. Publié en français sous le titre Ces corps qui comptent. De la matérialité et des limites discursives du «sexe ", traduit de l'anglais par Charlotte Nordmann, Paris, Éditions Amsterdam, 2009.

16. Note de la traductrice : ici le jeu de mots proposé par l'auteur est intraduisible car le « mot en $-\mathrm{p}$ » renverrait autant à la notion de performativité qu'au champ des " performing arts ", syntagme au pluriel que nous avons ici décidé de traduire par l'expression française d'" arts vivants ".

17. Shannon Jackson, Lines of Activity: Performance, Historiography, Hull-House Domesticity, Michigan, University of Michigan Press, 2000.

18. Shannon Jackson, Social Works: Performing Art, Supporting Publics, Londres et New York, Routledge, 2011.

19. La performance, d'une durée de huit heures, (dont le titre pourrait être traduit par « Nettoyage, pistes, entretien - dedans et dehors ») fut présentée le 22 juillet 1973 au Musée Wadsworth Atheneum à Hartford dans le Connecticut.

20. En français dans le texte.

21. Judith Butler, «Against Proper Objects. Introduction » dans Differences: A Journal of Feminist Cultural Studies, Duke University Press, 6.2-3, été/automne 1994, p. 6.

22. Elin Diamond, "Brechtian Theory/ Feminist Theory: Toward a Gestic Feminist Criticism », The Drama Review, MIT Press, vol. 32, n 1, printemps 1988, p. 82-94.

23. Bertolt Brecht, «Description succincte d'une nouvelle technique d'art dramatique produisant un effet de distanciation » (1940) dans L'Art du comédien, Écrits sur le théâtre, traduit par Jean Tailleur et Guy Delfel, Paris, L'Arche, 1999, p. 130.

24. Ibid., p. 143. Cité par Elin Diamond, Unmaking Mimesis: Essays on Feminism and Theatre, Routledge, Londres et New-York, 1997, p. 84.

25. Elin Diamond, "Brechtian Theory/ Feminist Theory: Toward a Gestic Feminist Criticism », op. cit., p. 85.

26. Ibid.

27. Ibid.

28. Sue-Ellen Case "Towards a Butch-Femme Aesthetic ", Discourse 11, hiver 19881989 (réédité in The Lesbian and Gay Studies Reader, éd. Abelove et al., New York, Routledge, 1993, p. 294-306).

29. Note de la traductrice : Ce jeu de mots est intraduisible en français, le terme anglais de «slash» désignant à la fois la barre oblique comme marque de ponctuation en typographie et un type de bar lesbien.

30. Sue-Ellen Case, op. cit., p. 283.

31. Ibid., p. 291.

32. Sue-Ellen Case, op. cit., p. 292. 


\section{Genre et arts vivants}

33. Note de la traductrice : le terme anglais d'outsider, difficilement traduisible dans ce contexte, est laissé tel quel dans le texte.

34. Judith Butler, Gender Trouble: Feminism and the Subversion of Identity, op. cit.

35. Judith Butler, Trouble dans le genre, Pour un féminisme de la subversion, op. cit., p. 59.

36. Ibid., p. 61.

37. Ibid., p. 275.

38. Sue-Ellen Case, The Domain-Matrix: Performing Lesbian at the End of Print Culture, Indiana, Indiana University Press,1996, p. 16.

39. Elin Diamond, «Brechtian Theory/ Feminist Theory: Toward a Gestic Feminist Criticism ", op. cit., p. 85.

40. Elin Diamond, Unmaking Mimesis: Essays on Feminism and Theatre, op. cit.

41. Elin Diamond, "Brechtian Theory/ Feminist Theory: Toward a Gestic Feminist Criticism ", op. cit., p. 85.

42. Elin Diamond, Unmaking Mimesis: Essays on Feminism and Theatre, op. cit., p. 46.

43. Ibid., p. 46-47. 